\title{
ON BINOMIAL UNITS OF CERTAIN CUBIC FIELD
}

\author{
KIYOTA OZEKI
}

\begin{abstract}
Scarowsky has conjectured about a binomial unit in a cubic field. We discuss a relation between binomial units and a diophantine equation.
\end{abstract}

In a recent paper, Scarowsky [3] has conjectured about a binomial unit in a cubic field. We prove the following

THEOREM. Let $K=Q(\theta)$ be a cubic field, where $\theta^{3}+12 a \theta-12=0, a>0$. If $\varepsilon=1-a \theta$ is the fundamental unit of $K$, it follows that all solutions of $x^{3}+12 a x y^{2}-$ $12 y^{3}=1$ are $(x, y)=(1,0),(1, a)$.

LEMMA 1 [1]. If $b \theta+c$, where $b \neq 0, \pm 1$, is a positive unit in $Z[\theta]$ where $\theta^{3}-P \theta^{2}+Q \theta-R=0$, then no power $>1$ of $b \theta+c$ can be a binomial unit.

LeMma 2 [2]. Let $\varepsilon= \pm \theta+c$ be a unit in $Z[\theta]$, where $\theta^{3}-P \theta^{2}+Q \theta-R=0$. If $\theta^{3} \equiv 0\left(\bmod p^{2}\right)$, where $p$ is a prime, then $p+c$ and $\varepsilon^{n}=u+v \theta$ is impossible for $n>1$.

The Diophantine equation $x^{3}+12 a x y^{2}-12 y^{3}=1$ can be written as a norm equation

$$
N_{K / Q}(x-\theta y)=1
$$

All solutions of the Diophantine equation are given by $x-\theta y=(1-a \theta)^{n}$, $n \in Z$.

Using Lemma 1 and 2, it is sufficient to prove that $\varepsilon^{-n}$ cannot be a binomial unit for $n \in N$. It is easy to calculate an inverse unit $\varepsilon^{-1}, \varepsilon^{-1}=\left(1+12 a^{3}\right)+a \theta+a^{2} \theta^{2}$.

Put $\varepsilon^{-n}=\alpha_{n}+\beta_{n} \theta+\gamma_{n} \theta^{2}$, where $\alpha_{0}=1, \beta_{0}=0, \gamma_{0}=0, \alpha_{1}=1+12 a^{3}, \beta_{1}=a$, $\gamma_{1}=a^{2}$

\section{LEMMA 3.}

$$
\left(\alpha_{n}, \beta_{n}, \gamma_{n}\right)=(1,0,0) M^{n}(a), \quad M(a)=\left(\begin{array}{ccc}
1+12 a^{3} & 12 a^{2} & 12 a \\
a & 1 & 0 \\
a^{2} & a & 1
\end{array}\right)
$$

Received by the editors October 11, 1985.

1980 Mathematics Subject Classification (1985 Revision). Primary 12A30.

1986 American Mathematical Society $0002-9939 / 86 \$ 1.00+\$ .25$ per page 
Proof. Calculating $\varepsilon^{-(n+1)}$, we have

$$
\begin{aligned}
\varepsilon^{-(n+1)} & =\varepsilon^{-1} \varepsilon^{-n} \\
& =\left\{\left(1+12 a^{3}\right)+a \theta+a^{2} \theta^{2}\right\}\left(\alpha_{n}+\beta_{n} \theta+\gamma_{n} \theta^{2}\right) \\
& =\left(a^{2} \alpha_{n}+a \beta_{n}+\gamma_{n}\right) \theta^{2}+\left(a \alpha_{n}+\beta_{n}\right) \theta+\left(1+12 a^{3}\right) \alpha_{n}+12 a^{2} \beta_{n}+12 a \gamma_{n} .
\end{aligned}
$$

Comparing coefficients of $\theta, \theta^{2}$ and constant term, we obtain

$$
\begin{aligned}
& \alpha_{n+1}=\left(1+12 a^{3}\right) \alpha_{n}+12 a^{2} \beta_{n}+12 a \gamma_{n}, \\
& \beta_{n+1}=a \alpha_{n}+\beta_{n}, \\
& \gamma_{n+1}=a^{2} \alpha_{n}+a \beta_{n}+\gamma_{n},
\end{aligned}
$$

or in matrix expression, $\left(\alpha_{n+1}, \beta_{n+1}, \gamma_{n+1}\right)=\left(\alpha_{n}, \beta_{n}, \gamma_{n}\right) M(a)$. Therefore,

$$
\left(\alpha_{n+1}, \beta_{n+1}, \gamma_{n+1}\right)=(1,0,0) M^{n+1}(a) \text {. }
$$

Proof of The Theorem. Generally we have to calculate $M^{n}(a)$ explicitly, using, for example, the Jordan canonical form of $M(a)$. However, in our case, it is enough to check that $\alpha_{n}, \beta_{n}, \gamma_{n} \neq 0$ for $n \geqslant 1$. All entries of the first column of $M^{n}(a)$ are greater than $1+12 a^{3}$ for $n \geqslant 2$, so we obtain $\alpha_{n}, \beta_{n}, \gamma_{n}>0$.

\section{REFERENCES}

1. B. N. Delone and D. K. Faddeev, The theory of irrationalities of the third degree, Transl. Math. Monos., vol. 10, Amer. Math. Soc., Providence, R. I., 1964.

2. B. Gordon and S. P. Mohanty, On a theorem of Delaunay and some related results, Pacific J. Math. 68 (1977), 399-409.

3. M. Scarowsky, On units of certain cubic fields and the diophantine equation, $x^{3}+y^{3}+z^{3}=3$, Proc. Amer. Math. Soc. 91 (1984), 351-356.

Department of Mathematics, The Faculty of General Education, Utsunomiya University, 350 MiNE-MACHI, UTSUNOMIYA, JAPAN 